\title{
ULTRASOUND PROVEN MONOSODIUM URATE CRYSTAL DEPOSITS IN THE JOINTS ARE ASSOCIATED WITH SMALLER KIDNEY SIZE, DECREASED INTRARENAL BLOOD FLOW AND ARTERIOSCLEROTIC TYPE VASCULAR CHANGES
}

\author{
R. Gancheva', T. Kundurzhiev², Zl. Kolarov ${ }^{1}$, A. Koundurdjiev ${ }^{3}$ \\ ${ }^{1}$ Clinic of Rheumatology, University Hospital Sv. Iv. Rilski - Sofia, Bulgaria \\ ${ }^{2}$ Faculty of Public Health, Medical University - Sofia, Bulgaria \\ ${ }^{3}$ Clinic of Nephrology, University Hospital Sv. Iv. Rilski, Medical University - Sofia, Bulgaria
}

\begin{abstract}
Objective: To establish the association between ultrasound (US) burden with articular MSU crystals and renal morphology, blood supply, function and arteriosclerotic carotid arteries alterations in gout patients, individuals with asymptomatic hyperuricemia and no sign of inflammatory arthritis and psoriatic arthritis subjects with asymptomatic hyperuricemia. Methods: 121 consecutive patients were included: 85 patients with gout, 27 subjects with asymptomatic hyperuricemia and 9 psoriatic arthritis patients. Subjects underwent US of both kidneys, common carotid arteries and bilateral US of the joints of the hands, elbows, knees, ankles and feet. For intrarenal blood flow we judged by measuring the renal resistive index (RRI). By US of the carotid arteries were determined intima-media thickness (IMT), common carotid artery resistive index (CCARI) and the presence of atherosclerotic plaques was registered. Results: Individuals with articular US MSU deposits compared to those without had higher RRI $(p=0.035)$ and smaller kidney size $(p=0.014)$, but renal parenchymal thickness $(p=0.893)$, echogenicity $(p=$ $0.291), I M T(p=0.165), C C A R I(p=0.097)$, the frequency of nephrolithiasis $(p=0.438)$ and atherosclerotic plaques $(p=0.830)$ were similar. Subjects with US evidence of MSU crystals in two or more joint regions had the highest RRI $(p=0.002)$ and CCARI $(p=$ 0.019). Compared to gout patients the risk of MSU crystal accumulation in the joints of asymptomatic hyperuricemia group was lower by $82.7 \%$, OR $=0.173(95 \% \mathrm{Cl} ; 0.060-$ $0.498, p=0.001$ ), while in psoriatic arthritis patients the risk was lower by $82 \%$, OR = 0.180 (95\% Cl; 0.038-0.861, $p=0.032$ ). Conclusions: This study points out that subjects with larger extent of articular MSU burden have greater vascular stiffness. The accumulation of MSU crystals in the joints may be associated with the accumulation of crystals mainly in the renal interstitium.
\end{abstract}

Key words: US MSU crystal deposits, kidney size, intrarenal blood flow, carotid arteries stiffness

Corresponding author: Rada Gancheva MD, PhD, Clinic of Rheumatology, University Hospital Sv. Iv. Rilski, Sofia, Bulgaria 


\section{INTRODUCTION}

$\mathrm{t}$ is not yet clear whether articular monosodium urate (MSU) crystal deposits in gout patients proven by ultrasound (US) are related to an increased risk of cardiovascular complications. Data whether these deposits are connected to impaired renal function, changes in renal blood flow, nephrolithiasis and arteriosclerotic vascular alterations, in patients in the spectrum of gout as well as in individuals who have inflammatory arthritis with accompanying asymptomatic hyperuricemia, are insufficient.

Hyperuricemia is a frequent finding in psoriasis and psoriatic arthritis [1], which may be linked to the increased cell turnover and the release of proinflammatory cytokines and tumor necrosis factor [2]. Gout and psoriatic arthritis share common risk factors. Serum uric acid in psoriatic arthritis patients is not connected to the extent of skin involvement but reflects metabolic changes, especially hypercholesterolemia and renal impairment [3]. In 2015 Merola using a huge database from the Health Professionals Follow-up study (19862010) and Nurses' Health Study (1998-2010) demonstrated that psoriasis and psoriatic arthritis were associated with an increased risk of gout [4]. On the other hand, studies report that MSU crystals could play a pathogenic role for the development of psoriatic arthritis [5]. Tsuruta et al. revealed that hyperuricemia increases uric acid crystallization in and around joints of psoriatic patients. Given that MSU crystals are potent inducers of the innate immune response, this could be one of the mechanisms by which they trigger the development of psoriatic arthritis in psoriatic subjects [6].

In the present study we aimed to establish the association between US burden with MSU crystals in the joints and renal morphology, blood supply, function and arteriosclerotic common carotid arteries alterations in gout patients, individuals with asymptomatic hyperuricemia and no sign of inflammatory arthritis and psoriatic arthritis subjects with asymptomatic hyperuricemia. In order to achieve our aim we applied a complex multimodal ultrasonography which is painless, noninvasive, allows multiplanar target assessment and is characterized with no exposure to ionizing radiation.

\section{PATIENTS AND METHODS}

This was a single center cross-sectional study, including 121 consecutive patients divided into 85 patients with gout, 27 subjects with asymptomatic hyperuricemia and no sign of inflammatory arthritis and 9 psoriatic arthritis individuals with accompanying asymptomatic hyperuricemia (demographic, clinical, laboratory and US characteristics of the kidneys and common carotid arteries in the three groups are summarized in table 1).

Gout patients fulfilled the 2015 American College Classification criteria [7] and had no subcutaneous tophi. Some of the patients, mainly those with asymptomatic hyperuricemia attended the outpatient clinic, while the others were hospitalized for examinations regarding their further treatment. At enrollment patients were physically examined by a rheumatologist from the Rheumatology Clinic of the University Hospital "Sv. Iv. Rilski" in Sofia, Bulgaria. Study protocol has been approved by the ethics committee of the institution. All participants gave informed consent in accordance with the Declaration of Helsinki.

Clinical and laboratory assessments included collecting detailed information related to current smoking habits, presence of arterial hypertension defined as systolic blood pressure $\geq 140 \mathrm{~mm} \mathrm{Hg}$, diastolic blood pressure $\geq 90 \mathrm{~mm} \mathrm{Hg}$ or use of antihypertensive medication; diabetes mellitus, based on physician diagnosis and/or documented use of insulin and/or oral hypoglycemic agents; dyslipidemia, based on elevated fasting lipid levels or low HDL and/or documented use of lipid-lowering agents; reduced glomerular filtration rate taken at eGFR $<90 \mathrm{ml} / \mathrm{min}$ calculated by the Cockroft-Gault formula. The other laboratory parameters considered in the analysis were serum uric acid, creatinine, blood urea nitrogen (BUN). Hyperuricemia was defined as serum level more than $360 \mu \mathrm{mol} / \mathrm{l}$ for women and more than $400 \mu \mathrm{mol} / \mathrm{l}$ for men. Laboratory records of men with serum uric acid levels less than $400 \mu \mathrm{mol} / \mathrm{l}$ were reviewed, and if hyperuricemia was present in the previous year, they were also enrolled. Patients were defined as having suffered a cardiovascular event if they had experienced a myocardial infarction, stroke and/or occlusion of a peripheral artery. Exclusion criteria were exacerbated cardiac or renal failure, cardiomyopathy, severe valvulopathy, arterial blood pressure $>140 / 90 \mathrm{~mm} \mathrm{Hg}$ and heart rate $<50$ bpm or $>90$ bpm during the US measurements of the kidneys and common carotid arteries.

All patients underwent bilateral US examination of distal interphalangeal, proximal interphalangeal, metacarpophalangeal joints, midcarpal joint, radiocarpal joint together with the six extensor compartments of the wrist; elbow joint and triceps tendon insertion; knee joint together with quadriceps and patellar tendon (both proximal and distal) insertions; tibiotalar joint with peroneous longus and brevis; tibialis posterior and Achilles tendon insertion; talonavicular joint and the five metatarsophalangeal joints. US measurements were conducted on Esaote-Mylab Twice with a high-frequency linear 
transducer 4-15 MHz, by one trained ultrasonographer, who was aware of the clinical and laboratory characteristics of the patients. All regions were evaluated by standard positions [8] covering all parts of the joints and tendons by sweeping the regions. The existence of double contour sign, intra-tendinous MSU aggregates, "snow storm", tophi, tophi with erosions, or a combination of these US features was assessed. In each of the examined joint regions of each patient the presence of the particular US MSU finding or the absence of MSU crystal deposits was registered.

Further, all subjects underwent US of the kidneys and common carotid arteries performed on Sonoscape medical corp. model S22, by another certified operator, who was blinded to the cases clinical and laboratory data. Kidneys were examined with $3.5 \mathrm{MHz}$ transducer, working with pulse Doppler frequency of $2.5 \mathrm{MHz}$. Renal length, parenchymal thickness and echogenicity were determined bilaterally. The presence of nephrolithiasis was reported. By means of the value of renal resistive index (RRI) we judged for intrarenal blood flow [9]. The parameter was determined in both kidneys at the level of interlobar arteries. The Doppler probe volume was $2 \mathrm{~mm}$ with pulse repetition frequency from 1.5 to $2 \mathrm{kHz}$ and angle of orientation $<60^{\circ}$. Carotid arteries were examined with $10 \mathrm{MHz}$ linear transducer working with pulse Doppler frequency of $5 \mathrm{MHz}$. Intima-media thickness (IMT) was bilaterally recorded in the far wall of the common carotid arteries $1-2 \mathrm{~cm}$ proximal to the bifurcation. Common carotid artery resistive index (CCARI), which reflects the stiffness of the common carotid arteries, was determined with a probe volume of pulse Doppler $3 \mathrm{~mm}$ and angle of orientation between $30^{\circ}$ and $60^{\circ}[10,11]$. The presence of heterogeneous or homogeneous atherosclerotic plaques was registered.

Our outcome variables were articular US MSU crystal deposits, kidney size, RRI, CCARI and atherosclerotic plaques. Explanatory variables were the clinical and laboratory characteristics of the patients.

Table 1. Demographic, clinical, laboratory and US characteristics of the kidneys and common carotid arteries in the three groups

\begin{tabular}{|c|c|c|c|c|c|}
\hline Indices & Gout patients & $\begin{array}{l}\text { Asymptomatic hy- } \\
\text { peruricemia patients }\end{array}$ & $\begin{array}{l}\text { Psoriatic arthritis patients with } \\
\text { asymptomatic hyperuricemia }\end{array}$ & \multicolumn{2}{|c|}{ p-value } \\
\hline Age (years), Mean (SD) & $57.76(14.11)$ & $53.44(17.21)$ & $61.22(14.42)$ & 0.387 & $\mathrm{~K}$ \\
\hline Sex (males), n (\%) & $63(74.1) a$ & $13(48.1) b$ & $2(22.2) b$ & 0.001 & $E$ \\
\hline BMI $\left(\mathrm{kg} / \mathrm{m}^{2}\right)$, Mean $(\mathrm{SD})$ & $29.35(4.35)$ & $29.80(5.51)$ & $30.64(4.42)$ & 0.735 & $\mathrm{~K}$ \\
\hline Serum uric acid $(\mu \mathrm{mol} / \mathrm{l})$, Mean $(\mathrm{SD})$ & $480.96(102.89)$ & $460.97(90.90)$ & $414.56(46.76)$ & 0.071 & $\mathrm{~K}$ \\
\hline BUN (mmol/l), Mean (SD) & $6.75(3.34)$ & $6.24(2.22)$ & $6.51(2.62)$ & 0.895 & $\mathrm{~K}$ \\
\hline eGFR (ml/min), Mean (SD) & $95.74(36.09)$ & $102.03(36.26)$ & $99.66(32.88)$ & 0.775 & $\mathrm{~K}$ \\
\hline Dyslipidemia, n (\%) & $37(92.5)$ & $14(56.0)$ & $6(100.0)$ & 1.000 & $E$ \\
\hline BMI $(>30$ kg/m²), n (\%) & $37(43.5)$ & $15(48.1)$ & $5(55.6)$ & 0.757 & $E$ \\
\hline Arterial hypertension, $\mathrm{n}(\%)$ & $65(76.5)$ & $16(59.3)$ & $6(66.7)$ & 0.208 & $E$ \\
\hline Patients suffered a cardiovascular event, $\mathrm{n}(\%)$ & $13(15.3)$ & $3(11.1)$ & $1(11.1)$ & 0.906 & $E$ \\
\hline Patients with diabetes mellitus, $\mathrm{n}(\%)$ & $13(15.3)$ & $5(18.5)$ & $1(11.1)$ & 0.913 & $E$ \\
\hline Patients with chronic kidney disease, $\mathrm{n}(\%)$ & $36(45.6)$ & $11(44.0)$ & $4(44.4)$ & 1.000 & $\mathrm{E}$ \\
\hline Smokers, n (\%) & $23(27.4)$ & $9(33.3)$ & $3(33.3)$ & 0.806 & $E$ \\
\hline \multicolumn{6}{|l|}{ Atherosclerotic plaques, $\mathrm{n}(\%)$} \\
\hline None & $49(57.6)$ & $13(48.1)$ & $4(44.4)$ & 0.595 & $E$ \\
\hline Heterogeneous plaques & $19(22.4)$ & $7(25.9)$ & $4(44.4)$ & & \\
\hline Homogeneous plaques & $17(20.0)$ & $7(25.9)$ & $1(11.1)$ & & \\
\hline Patients treated with diuretics, $\mathrm{n}(\%)$ & $57(67.1)$ & $19(70.4)$ & $6(66.7)$ & 0.948 & $E$ \\
\hline Patients treated with allopurinol, $\mathrm{n}(\%)$ & $10(11.8)$ & $2(7.4)$ & $0(0.0)$ & 0.678 & $E$ \\
\hline Patients treated with febuxostat, $\mathrm{n}(\%)$ & $19(22.4) \mathrm{a}$ & $0(0.0) \mathrm{b}$ & $0(0.0) \mathrm{b}$ & 0.005 & $E$ \\
\hline Patients treated with benzbromarone, $\mathrm{n}(\%)$ & $5(5.9)$ & $1(3.7)$ & $0(0.0)$ & 1.000 & $E$ \\
\hline NSAIDs, n (\%) & $29(40.3) a$ & $1(3.8) \mathrm{b}$ & $2(22.2) a, b$ & 0.001 & $E$ \\
\hline Patients with nephrolithiasis, $\mathrm{n}(\%)$ & $14(16.9)$ & $4(14.8)$ & $1(11.1)$ & 0.889 & $E$ \\
\hline Kidney size, Mean (SD) & $54.48(5.44) \mathrm{a}$ & 56.63 (5.66)a.b & $58.04(4.29) \mathrm{b}$ & 0.046 & $\mathrm{~K}$ \\
\hline RRI, Mean (SD) & $0.69(0.06)$ & $0.66(0.08)$ & $0.70(0.06)$ & 0.075 & $\mathrm{~K}$ \\
\hline IMT, Mean (SD) & $0.92(0.19)$ & $0.86(0.23)$ & $0.99(0.27)$ & 0.260 & $\mathrm{~K}$ \\
\hline CCARI, Mean (SD) & $0.70(0.06)$ & $0.68(0.07)$ & $0.73(0.09)$ & 0.089 & $\mathrm{~K}$ \\
\hline
\end{tabular}

Abbr.: BMI - body mass index; BUN - blood urea nitrogen; eGFR - estimated glomerular filtration rate; NSAIDs - nonsteroidal anti-inflammatory drugs; RRI - renal resistive index; IMT - intima-media thickness; CCARI - common carotid artery resistive index; K-Kruskal Wallis test; E-Fisher's exact test. Note: The presence of identical superscript between different groups demonstrates the lack of significant difference in the mean values of the examined parameter ( $p>0.05)$. Different superscripts indicate a significant difference in the mean values of the examined parameter $(p<0.05)$. The absence of superscripts in the intergroup comparisons means no significant differences in the mean values. 


\section{STATISTICAL ANALYSES}

Statistical analyses were done by using SPSS version 13.0 (SPSS Inc., Chicago, IL, USA). Means with SDs and percentages were calculated to describe the clinical and US characteristics of the patients. Comparison of the relative shares of the categorical variables was performed by Chi-Square or Fisher's exact test. Among two independent groups comparisons of the quantitative variables with normal distribution were done by the t-test, when the distribution was not normal the test of Mann-Whitney was used. In the normal distribution comparisons between more than two groups were conducted by ANOVA followed by the Tukey HSD Post Hoc Test, and when the distributions were not normal the test of Kruskal Wallis was applied. Factors for the accumulation of MSU crystals were studied by multivariate binary logistic regression. All tests were two tailed, and $p$ values less than 0.05 were considered statistically significant.

\section{RESULTS}

Patients in the study were age-matched. The distribution of known cardiovascular risk factors was similar among the groups. Kidney size was the smallest in gout sub- jects but no significant difference was registered with asymptomatic hyperuricemia individuals. The mean values of RRI as well as the distribution of nephrolithiasis were comparable among the groups. The same applies to the mean values of IMT $(p=0.260)$, CCARI $(p=0.089)$ and the frequency of heterogeneous or homogeneous carotid arteries plaques $(p=0.595)$, (Table 1$)$.

The distribution of MSU crystal deposits in two or more joint regions was the highest in gout patients (56.5\%) compared to patients with asymptomatic hyperuricemia $(11.1 \%)$ and individuals with psoriatic arthritis $(22.2 \%),(p<0.001)$. When patients were divided according to the presence of articular MSU crystals, we observed that the frequency of chronic kidney disease $(p=0.534)$ and nephrolithiasis $(p=0.438)$ was equal in both subdivisions (Fig. $1 \mathrm{a}, \mathrm{b}$ ). However, in individuals with US evidence of articular MSU crystals RRI was higher $(p=0.035)$ and kidneys were smaller $(p$ $=0.014)$ but renal parenchymal thickness $(p=0.893)$, echogenicity ( $p=0.291$ ) (Fig. $2 a, b, c, d)$, as well as the distribution of patients treated with NSAIDs $(p=$ 0.236) were comparable (Fig. 1c). As regards the common carotid arteries the means of IMT $(p=0.165)$,

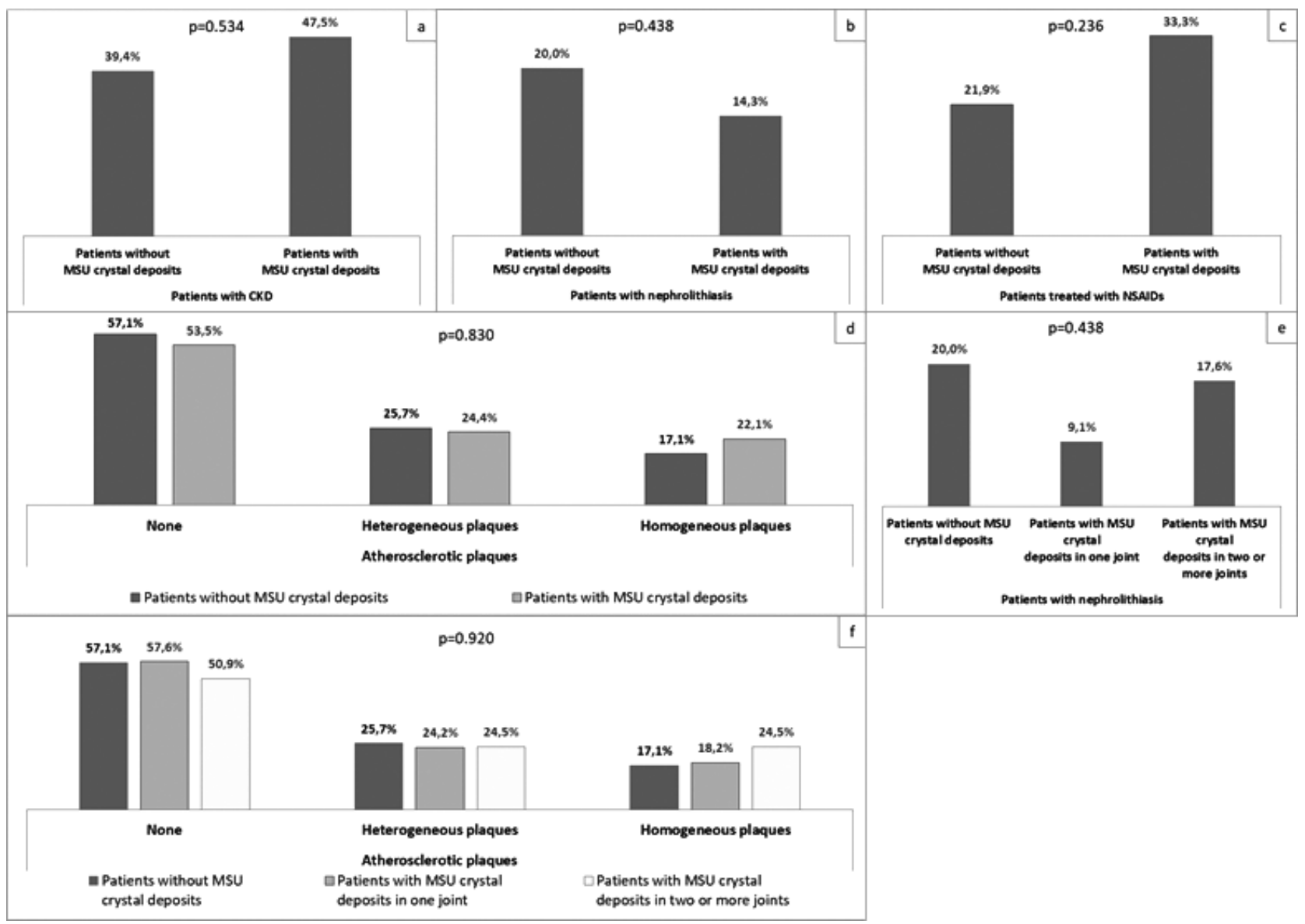

Fig. 1. Distribution of the patients by the presence of chronic kidney disease (CKD), nephrolithiasis, treatment with nonsteroidal antiinflammatory drugs (NSAIDs), heterogeneous or homogeneous atherosclerotic plaques and MSU crystal deposits in the joint regions (a, $b, c, d)$. Distribution of the patients by the presence of CKD, nephrolithiasis, heterogeneous or homogeneous atherosclerotic plaques and MSU crystal deposits in one or more joint regions (e, f, g) 

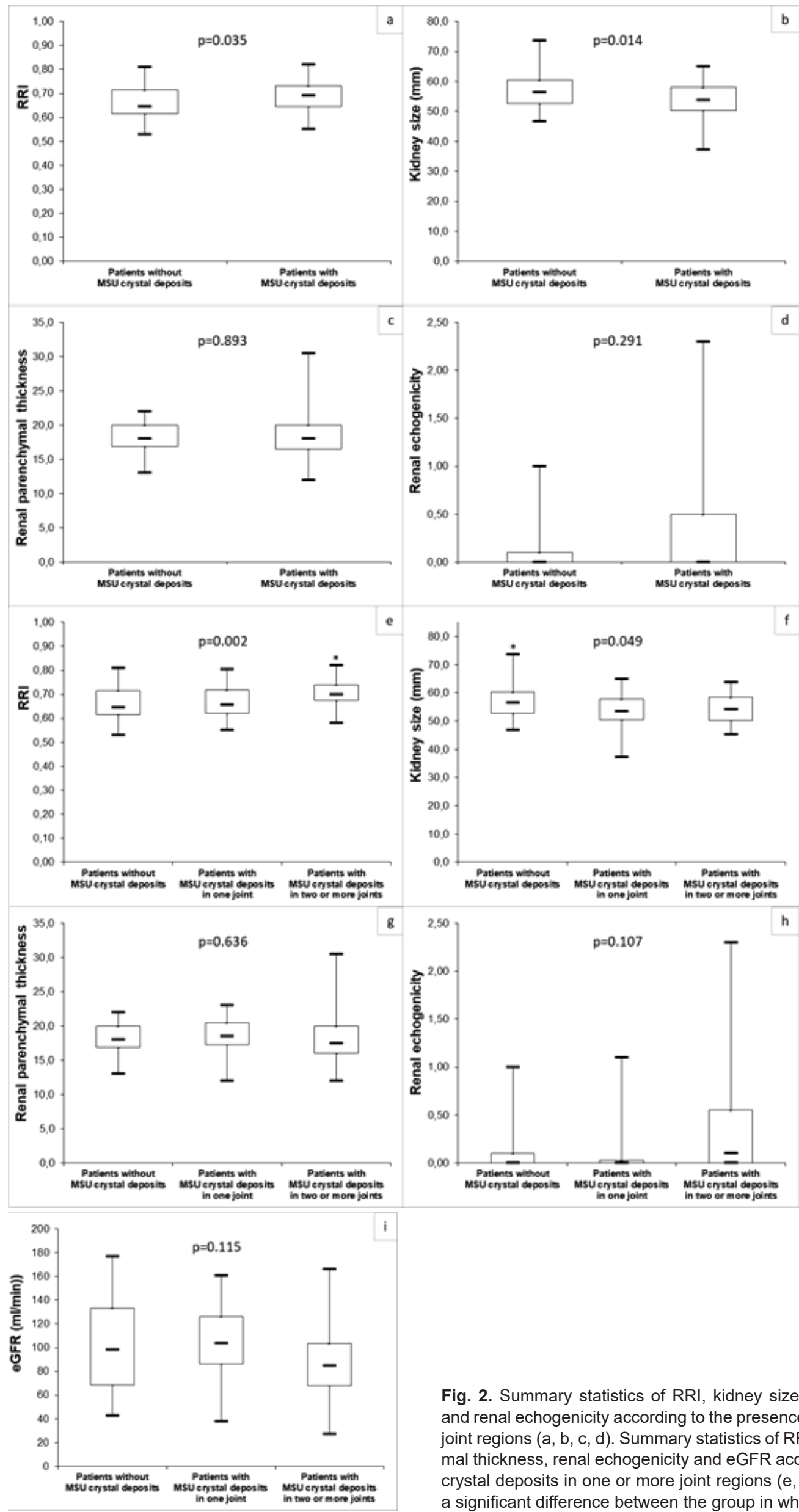

Fig. 2. Summary statistics of RRI, kidney size, renal parenchymal thickness and renal echogenicity according to the presence of MSU crystal deposits in the joint regions (a, b, c, d). Summary statistics of RRI, kidney size, renal parenchymal thickness, renal echogenicity and eGFR according to the presence of MSU crystal deposits in one or more joint regions (e, f, g, h, i). (Note: $\left(^{*}\right)$ - indicates a significant difference between the group in which it is with the other groups) 


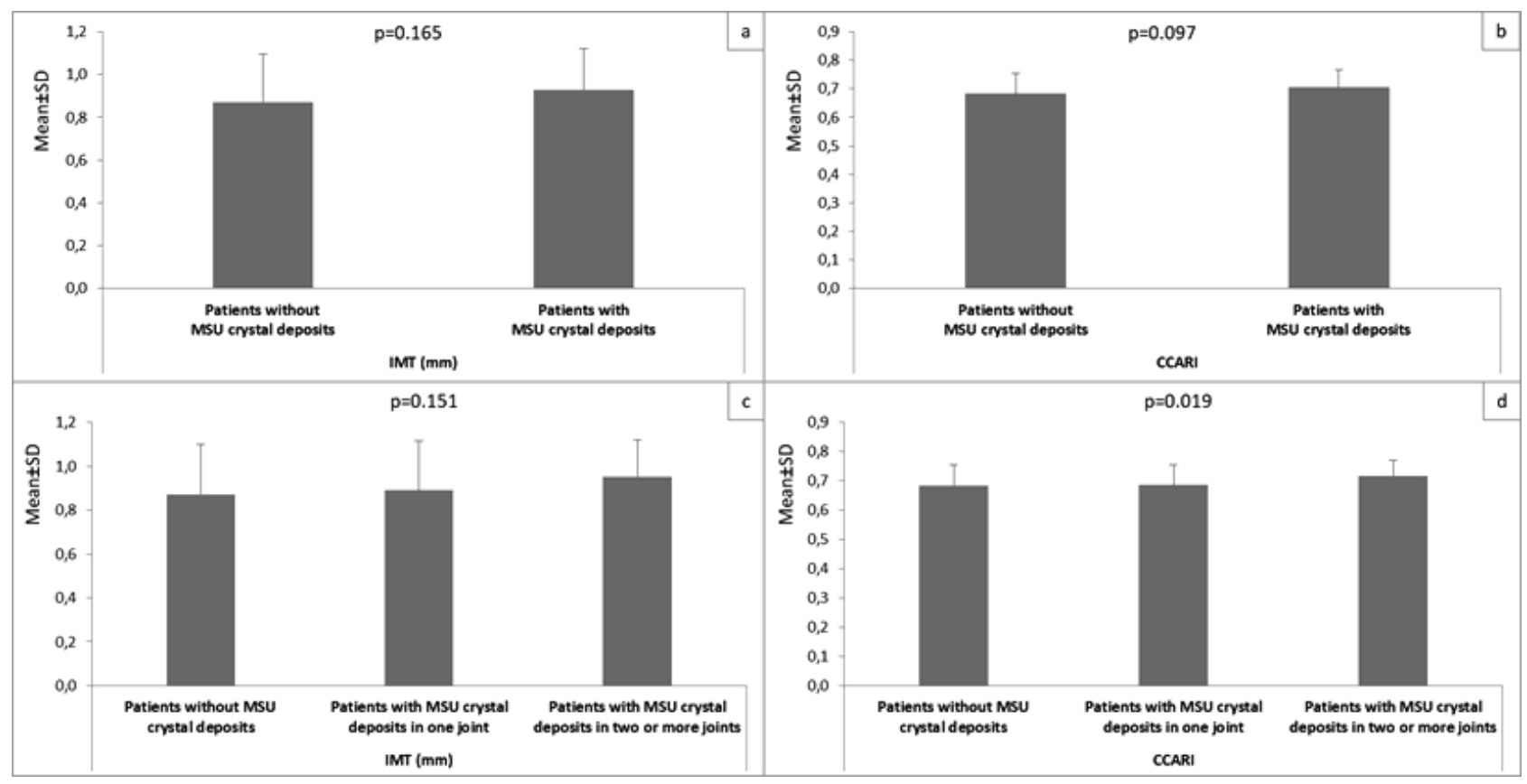

Fig. 3. Summary statistics of IMT and CCARI according to the presence of MSU crystal deposits in the joint regions (a, b). Summary statistics of IMT and CCARI according to the presence of MSU crystal deposits in one or more joint regions (c, d)

CCARI $(p=0.097)$ (Fig. $3 a, b)$ and the frequency of heterogeneous or homogeneous plaques $(p=0.830)$ (Fig. 1d) remained unchanged in subjects without and with evidence of MSU crystals in the joints. Characteristics of the patients without and with MSU crystal deposits in the joints are presented in table 2.

Further, patients with MSU crystal deposits in two or more joint regions, compared to those who had de- posits in only one joint region and patients without US evidence of MSU crystals, had the highest RRI ( $p$ $=0.002$ ) but the mean values of renal parenchymal thickness $(p=0.636)$, echogenicity $(p=0.107)$, eGFR $(p=0.115)$ and frequency of nephrolithiasis $(p=0.438)$ were similar (Fig. 2 e, g, h, i and Fig.1 e). Among subjects with US MSU deposits in one joint region and those who had larger extent of MSU deposits there

Table 2. Demographic, clinical, laboratory and US characteristics of the kidneys and common carotid arteries in patients without crystal deposits in the joints and subjects with MSU crystal deposits in the joints

\begin{tabular}{|c|c|c|c|}
\hline Indices & Patients without MSU crystal deposits & Patients with MSU crystal deposits & $p$-value \\
\hline Age (years), Mean (SD) & $54(16.5)$ & $58(14)$ & 0.147 \\
\hline Sex (males), $\mathrm{n}(\%)$ & $20(57.1)$ & $58(67.4)$ & 0.283 \\
\hline $\mathrm{BMI}\left(\mathrm{kg} / \mathrm{m}^{2}\right)$, Mean (SD) & $29.03(4.96)$ & $29.76(4.45)$ & 0.432 \\
\hline Serum uric acid $(\mu \mathrm{mol} / \mathrm{l})$, Mean $(\mathrm{SD})$ & $454(69.07)$ & $479(107.6)$ & 0.211 \\
\hline BUN (mmol/l), Mean (SD) & $7.14(3.55)$ & $6.41(2.83)$ & 0.279 \\
\hline eGFR (ml/min), Mean (SD) & $103.38(40.77)$ & $95(33.33)$ & 0.258 \\
\hline Dyslipidemia, n (\%) & $18(90 \%)$ & $39(95.1 \%)$ & 0.591 \\
\hline $\mathrm{BMI}\left(>30 \mathrm{~kg} / \mathrm{m}^{2}\right), \mathrm{n}(\%)$ & $14(40)$ & $41(47.7)$ & 0.442 \\
\hline Arterial hypertension, $\mathrm{n}(\%)$ & $23(65.7)$ & $64(74.4)$ & 0.334 \\
\hline Patients suffered a cardiovascular event, n (\%) & $4(11.4)$ & $13(15.1)$ & 0.775 \\
\hline Patients with diabetes mellitus, $\mathrm{n}(\%)$ & $6(17.1)$ & $13(15.1)$ & 0.781 \\
\hline Patients with chronic kidney disease, $n(\%)$ & $13(39.4)$ & $38(47.5)$ & 0.431 \\
\hline Smokers, n (\%) & $10(28.6)$ & $25(29.4)$ & 0.927 \\
\hline \multicolumn{4}{|l|}{ Atherosclerotic plaques, $\mathrm{n}(\%)$} \\
\hline None & $20(57.1)$ & $46(53.5)$ & \\
\hline Heterogeneous plaques & $9(25.7)$ & $21(24.4)$ & 0.830 \\
\hline Homogeneous plaques & $6(17.1)$ & $19(22.1)$ & \\
\hline Patients treated with diuretics, $\mathrm{n}(\%)$ & $10(28.6)$ & $29(33.7)$ & 0.583 \\
\hline Patients treated with allopurinol, $\mathrm{n}(\%)$ & $1(2.9)$ & $11(12.8)$ & 0.176 \\
\hline Patients treated with febuxostat, $\mathrm{n}(\%)$ & $1(2.9)$ & $18(20.9)$ & 0.013 \\
\hline Patients treated with benzbromarone, $\mathrm{n}(\%)$ & $2(5.7)$ & $4(4.7)$ & 1.000 \\
\hline NSAIDs, $\mathrm{n}(\%)$ & $7(21.9)$ & $25(33.3)$ & 0.236 \\
\hline Patients with nephrolithiasis, $\mathrm{n}(\%)$ & $7(20)$ & $12(14.3)$ & 0.438 \\
\hline Kidney size, Mean (SD) & $57.14(5.94)$ & $54.44(5.14)$ & 0.014 \\
\hline RRI, Mean (SD) & $0.66(0.07)$ & $0.69(0.06)$ & 0.035 \\
\hline IMT, Mean (SD) & $0.87(0.23)$ & $0.93(0.19)$ & 0.165 \\
\hline CCARI, Mean (SD) & $0.68(0.07)$ & $0.71(0.06)$ & 0.097 \\
\hline
\end{tabular}


was no difference in renal length ( $p=0.979$ ), (Fig. 2 f). Furthermore, patients with MSU crystal deposits in two or more joint regions had the highest CCARI (mean \pm SD; $0.72 \pm 0.05$ vs $0.69 \pm 0.07$ vs $0.68 \pm 0.07$, $p=0.019)$ but the means of IMT $(p=0.151)$ and the distribution of atherosclerotic plaques $(p=0.920)$ were comparable (Fig. 3 c, d and Fig 1 f). Finally, a multiple logistic regression was conducted by which we estimated that compared to gout subjects the risk of MSU crystal accumulation in the joints of asymptomatic hyperuricemia group was lower by $82.7 \%$, OR $=0.173$ (95\% Cl; 0.060-0.498, $p=0.001)$, while in psoriatic arthritis individuals the risk was lower by $82 \%$, OR = 0.180 (95\% Cl; 0.038-0.861, $\mathrm{p}=0.032)$.

\section{DISCUSSION}

The main findings in this study were that patients with US evidence of MSU crystal deposits in the joints compared to those without MSU deposits in the joints had smaller kidney size and reduced intrarenal blood flow despite the lack of difference in renal parenchymal thickness, renal echogenicity and distribution of nephrolithiasis. Subjects with MSU crystal deposits in two or more joint regions had the most pronounced stiffness of the common carotid arteries.

We hypothesized that the smaller kidney size and the reduced intrarenal blood flow in individuals with US MSU crystals in the joints may be due to the accumulation of crystals in the renal interstitium. It has been proven that MSU crystals lead to the formation of intratubular precipitates causing tubular injury, interstitial inflammation and finally fibrosis. With the onset of fibrosis resistance of the renal vessels increases and blood flow significantly decreases [12, 13]. Also, it has been confirmed that MSU crystals can trigger necrosis of tubular cells via necroinflammation [14]. Judging indirectly from the US data, without having a biopsy finding, we consider our assumption possible. Bardin et al. established that hyperechoic deposits in the renal medulla of 503 Vietnamese gout patients correlated with deteriorated renal function and severe gout but were not linked to features of uric acid lithiasis [15]. We did not focus on hyperechoic deposits in the medulla, only the presence or absence of nephrolithiasis was registered. We examined three groups of patients, as one of them had inflammatory arthritis with accompanying asymptomatic hyperuricemia, with their total number being significantly smaller than that of the Vietnamese cohort.

Further, we observed that individuals with larger extent of MSU crystal deposits in the joints had the highest stiffness of the common carotid arteries with no significant change to be registered in the thick- ness of intima-media and in the frequency of heterogeneous or homogeneous plaques. This may be related to the predominantly arteriosclerotic type of vascular changes leading to increased vascular resistance rather than the atherosclerotic type, which is expressed by a thickened intima-media complex and the presence of plaques. In 2018 Andrés et al. reported that articular MSU deposits detected by US and confirmed by compensated polarized microscopy in asymptomatic hyperuricemia subjects suffering coronary event were connected to more advanced calcification at the coronary angiography [16]. In our study, we didn't have the opportunity to assess the degree of coronary arteries calcification. Later, in 2020 Calabuig et al. published that US power Doppler (PD) signal in newly diagnosed gout patients not taking urate lowering therapy was associated with the presence of atherosclerotic plaques. However, we did not analyze the PD signal, as we haven't found such a relationship [17]. By applying the multiple logistic regression analysis we estimated that the risk of MSU crystal accumulation in the joints of asymptomatic hyperuricemia patients and psoriatic arthritis subjects is comparable. These results could be due to the still incompletely studied and interrelated pathophysiological mechanisms between psoriatic arthritis, hyperuricemia and gout $[5,6]$.

In conclusion, the present study points out that individuals with US MSU crystal deposits in the joints have smaller kidney size and reduced intrarenal blood flow. The larger extent of articular US MSU burden is associated with advanced arteriosclerotic vascular alterations. To our knowledge, we are between the few who have tried to establish the relationship between US burden with articular MSU crystals and US damage of the kidneys and common carotid arteries.

We acknowledge that several limitations deserve to be mentioned. The first one is the cross-sectional design of the study. The second is the small number of patients in the psoriatic arthritis group. The third is that PD signal wasn't analyzed, which did not allow us to search for a connection with atherosclerotic plaques. Fourth, musculoskeletal US examinations were conducted by a researcher familiar with the clinical patients' data. We believe that the inclusion of renal biopsy in future similar studies would be beneficial. In this way our hypothesis that the accumulation of MSU crystals in the joints is associated with the accumulation of crystals mainly in the renal interstitium could be confirmed.

Disclosure summary: The authors have nothing to disclose. 


\section{REFERENCES:}

1. Lambert JR, Wright V. Serum uric acid levels in psoriatic arthritis. Ann Rheum Dis. 1977; 36:264-267.

2. Arevalo A, Haddadin F, Contreras G et al. Cardiovascular impact of hyperuricemia in patients with psoriatic arthritis [abstract]. Arth Rheum. 2019; 71 (suppl 10).

3. Bruce IN, Schentag CT, Gladman DD. Hyperuricemia in psoriatic arthritis. Journal of clinical rheumatology: a practical reports on rheumatic and musculoskeletal diseases 6(1):6-9.

4. Merola JF, Wu S, Han J et al. Psoriasis, psoriatic arthritis and risk of gout in US men and women. Ann Rheum Dis. 2015; 74(8):1495-1500.

5. Felten R, Duret PM, Gottenberg JE. At the crossroads of gout and psoriatic arthritis "psout". Clin Rheum, 2020; doi: 10.1007/s10067-020-04981-0.

6. Tsuruta N, Imafuku S, Narisawa Y. Hyperuricemia is an independent risk factor for psoriatic arthritis in psoriatic patients. $\mathrm{J}$ Dermatol. 2017; 44(12); 1349-1352.

7. Neogi T, Jansen TLT, Dalbeth $\mathrm{N}$ et al. Gout classification criteria. An American College of Rheumatology/European League Against Rheumatism Collaborative Initiative. Arth Rheum. 2015; 67:2557-2568.

8. Chizyński K, Rózycka M (2005). "Hyperuricemia". Pol. Merkur. Lekarski (in Polish). 19 (113): 693-6. PMID 16498814.

9. Backhaus M, Burmester GR, Gerber T, et al. Guidelines for musculoskeletal ultrasound in rheumatology. Ann Rheum Dis 2001; 60:641-9.

10. Viazzi F, Leoncini G, Derchi LE, Pontremoli R. Ultrasound Doppler renal resistive index: a useful tool for the management of the hypertensive patient. J Hypertens 2014; 32:149-153.
11. Staub D, Meyerhans A, Bundi B et al. Prediction of cardiovascular morbidity and mortality: comparison of the internal carotid artery resistive index with the common carotid artery intima-media thickness. Stroke 2006; 37:800-805.

12. Frauchinger B, Schmid HP, Roedel $\mathrm{C}$ et al. Comparison of carotid arterial resistive indices with intima-media thickness as sonographic markers of atherosclerosis.Stroke 2001; 32:836-841.

13. Mulay SR, Shi C, Ma X, Anders HJ. Novel insights into crystal-induced kidney injury. Kidney Dis 2018; 4:49-57.

14. Guerrot D, Dussaule, J, Kavvadas, $P$ et al. Progression of renal fibrosis: the underestimated role of endothelial alterations. Fibrogenesis Tissue Repair 2012; 5, S15.

15. Mulay SR, Holderied A, Kumar SV, Anders HJ. Targeting inflammation in so called acute kidney injury. Semin Nephrol 2016; 36:17-30.

16. Bardin T, Tran KM, Nguyen QD et al. Hyperechoic deposits in the renal medulla are associated with severe gout and decreased EGFR: a transversal study in 503 vietnamese patients. doi: 10.1136/annrheumdis-2018-eular.5027.

17. Andrés M, Quintanilla MA, Sivera F et al. Silent monosodium urate crystals deposits associate with severe coronary calcification in asymptomatic hyperuricemia: "An exploratory study”. Arth Rheum. doi 10.1002/art.39581.

18. Calabuig I, Martinez-Sanchis A, Andrés M. Carotid atherosclerosis and sonographic signs of urate crystal deposits in patients with gout: an association study. European Crystal Network 11 th Workshop 2020 p. 13 [abstract book].

Received: 18 June 2020, Accepted: 18 July 2020 